\title{
Heat Capacity, Resistivity, and Angular Dependent Magnetization Studies of Single Crystal $\mathrm{Nd}_{1+\epsilon} \mathrm{Fe}_{4} \mathrm{~B}_{4}$ for $\epsilon \approx \frac{1}{7}$
}

\author{
B. S. Conner ${ }^{\mathrm{a}}$, M. A. Susner ${ }^{\mathrm{a}}$, P. Lampen-Kelley, , A. F. May ${ }^{\mathrm{a}}$, M. A. McGuire ${ }^{\mathrm{a}}$, J. -Q. Yan ${ }^{\mathrm{a}}$, B. C. Sales ${ }^{\mathrm{a}}$ \\ ${ }^{a}$ Materials Science and Technology Division, Oak Ridge National Laboratory, Oak Ridge, Tennessee 37831 USA
}

\begin{abstract}
Advances in crystal growth have allowed for synthesis of large single crystals of $\mathrm{Nd}_{1+\epsilon} \mathrm{Fe}_{4} \mathrm{~B}_{4}$, a well-known phase with a modulated structure. As a result we are able to report heat capacity and resistivity measurements on a single crystal $\mathrm{Nd}_{1+\epsilon} \mathrm{Fe}_{4} \mathrm{~B}_{4}$ sample with a distribution of $\epsilon$ that skews towards the solubility limit of $\mathrm{Nd}$ near $\epsilon \approx \frac{1}{7}$. Heat capacity measurements show evidence of crystal field splitting at temperatures higher than the long-range ferromagnetic Curie temperature. Heat capacity, resistivity, and magnetization measurements all confirm a Curie temperature of $7 \mathrm{~K}$ which is lower than previously reported values in the $\mathrm{Nd}_{1+\epsilon} \mathrm{Fe}_{4} \mathrm{~B}_{4}$ system. We also perform measurements of the angular dependence of the magnetization and discover behavior associated with the magnetic anisotropy that is inconsistent with the simple description previously proposed .
\end{abstract}

Keywords:

$\mathrm{Nd}_{1+\epsilon} \mathrm{Fe}_{4} \mathrm{~B}_{4}$; magnetic anisotropy; crystal fields, resistivity, heat capacity

\section{Introduction}

A compound with a stoichiometry very close to $\mathrm{NdFe}_{4} \mathrm{~B}_{4}$ was discovered over thirty years ago as part of experiments designed to investigate ternary rare earthiron-boron systems [1], superstructures [2], and as a byproduct of experiments designed to study the effects of synthesis with excess neodymium and boron on coercivity in $\mathrm{Nd}_{2} \mathrm{Fe}_{14} \mathrm{~B}$ [3]. The nearly $\mathrm{NdFe}_{4} \mathrm{~B}_{4}$ compound was seen in [3] to exist as a prominent grain boundary phase in $\mathrm{Nd}_{2} \mathrm{Fe}_{14} \mathrm{~B}$ magnets. Like $\mathrm{Nd}_{2} \mathrm{Fe}_{14} \mathrm{~B}$, its structure was found to be essentially tetragonal. Various authors $[2,4,5,6,7,8]$ sythesized the purported

\footnotetext{
Current Address of M. A. Susner is UES Inc., Beavercreek, OH 45432 USA

Email address: connerbs@ornl.gov (B. S. Conner)

This manuscript has been authored by UT-Battelle, LLC under Contract No. DE-AC05-00OR22725 with the U.S. Department of Energy. The United States Government retains and the publisher, by accepting the article for publication, acknowledges that the United States Government retains a non-exclusive, paid-up, irrevocable, worldwide license to publish or reproduce the published form of this manuscript, or allow others to do so, for United States Government purposes. The Department of Energy will provide public access to these results of federally sponsored research in accordance with the DOE Public Access Plan (http://energy.gov/downloads/doepublic-access-plan).
}

Preprint submitted to Journal of Magnetism and Magnetic Materials
$\mathrm{NdFe}_{4} \mathrm{~B}_{4}$ under different conditions and, through structural studies using $\mathrm{X}$-ray diffraction (XRD), discovered that this phase could be more accurately described as $\mathrm{Nd}_{1+\epsilon} \mathrm{Fe}_{4} \mathrm{~B}_{4}$, where $\epsilon$ ranges from 0.09 to 0.142 . The toy-model structure has two tetragonal sub-lattices, one of iron and boron tetrahedra and one of neodymium atoms, that coexist with the same $a$ lattice parameter but with two distinct $c$ lattice parameters, here we call them $c_{n}$ and $c_{m}$ respectively. If the ratio $c_{m} / c_{n}$ is a simple fraction, here represented by the notation $m: n$, the structure is a commensurate superstructure. In the commensurate case the notation $m: n$ will consist only of integers. If $m: n$ cannot be written with integers then the structure is an incommensurate superstructure. The real structure of the $\mathrm{Nd}_{1+\epsilon} \mathrm{Fe}_{4} \mathrm{~B}_{4}$ phase is more complicated than the simple toy model, whether commensurate or incommensurate, for two reasons. One is that the FeB tetrahedra sub-lattice has periodic modulation $[9,7]$. Another is the fact that a structure with a single Nd:Fe-B $(m: n)$ layer ratio is not strictly observed on macroscopic length scales. A very local probe can, however, yield observations consistent with a definite $m: n$ layer ratio commensurate superstructure which was the case in reference [10] where the authors, using electron diffraction, found the maximum value of $\epsilon$ observed to date and what appeared to be a single, commensurate struc- 
ture through diffraction on a very thin sample. That value was $\epsilon=0.1429$ which corresponds to a layer ratio of $m: n=8: 7$ or similarly the compound $\mathrm{Nd}_{8} \mathrm{Fe}_{28} \mathrm{~B}_{28}$. However, the significant compositional variation in past reports leaves no reason for one to believe that a large $\mathrm{Nd}_{1+\epsilon} \mathrm{Fe}_{4} \mathrm{~B}_{4}$ sample, suitable for measurements of the physical properties, could possibly possess a single superstructure throughout its volume. This fact has been previously well established; see, for example, [7].

There are a number of phases in the $\mathrm{Nd}-\mathrm{Fe}-\mathrm{B}, \mathrm{Fe}-$ $\mathrm{B}$, and $\mathrm{Nd}-\mathrm{Fe}$ phase diagrams that are magnetic at room temperature and may manifest themselves as a magnetic impurity in $\mathrm{Nd}_{1+\epsilon} \mathrm{Fe}_{4} \mathrm{~B}_{4}$. However, $\mathrm{Nd}_{1+\epsilon} \mathrm{Fe}_{4} \mathrm{~B}_{4}$ itself has been shown to be non-magnetically ordered at room temperature $[3,11,5]$ and to show ferromagnetic ordering below either $10 \mathrm{~K}$ or $15 \mathrm{~K}[11,5]$, though CurieWeiss behavior at higher temperatures is shown only in [5] as those authors managed to either isolate a very pure sample or to subtract out the effects of impurities from the data presented. Detailed magnetization measurements are performed in [5] for samples with $\epsilon$ listed as $\approx \frac{1}{9}$ and at $4.2 \mathrm{~K}$ the magnetization vs. applied field, $M(H)$, measured along the crystallographic [ [ 110$]$ direction looks like a step-function, which is characteristic of a simple ferromagnet when measured with applied field parallel to one of its easy directions. It is worth defining the use of the term "easy direction" here. We use the term in the normal sense so that, for example, a uniaxial ferromagnet will be said to have two easy directions pointing opposite one another. We do not, however, use the term "easy direction" here to necessarily mean the direction of the easy axis as determined only by the magnetocrystalline anisotropy arising from the usual spin-orbit interaction in the magnetic atoms. We use it simply here to describe the observation of $M(\theta)$ maxima $(M(\theta)$ is parallel to the applied field) at fixed applied field magnitudes and changing angle $\theta$. See the experimental details section for more on this technique. More often than not there will be no distinction between these two definitions, especially if shape anisotropy effects can be eliminated, but a more complicated case probably arises for our $\mathrm{Nd}_{1+\epsilon} \mathrm{Fe}_{4} \mathrm{~B}_{4}$ experiments here so we choose to define the term "easy direction" as the result of an experimental observation. The case is made in [5], then, that there are four easy directions within the basal plane in their $\mathrm{Nd}_{1.11} \mathrm{Fe}_{4} \mathrm{~B}_{4}$ sample and that the basal plane is, itself, easy. They propose that those easy directions are the crystallographic [ $\left[\begin{array}{lll}1 & 1 & 0\end{array}\right],\left[\begin{array}{lll}-1 & 1 & 0\end{array}\right],\left[\begin{array}{ll}1 & -1\end{array}\right.$ $0]$, and [ $-1-10]$ directions. So, $M(H)$ very close to the [1 0 l 0 ] direction, for example, appears very much like a projection of the moment in the $\left[\begin{array}{lll}1 & 1 & 0\end{array}\right]$ direction onto the $\left[\begin{array}{lll}1 & 0 & 0\end{array}\right]$ direction. $M(H)$ in the $\left[\begin{array}{lll}0 & 0 & 1\end{array}\right]$ direction was also reported by those authors and is extremely small up to $7 \mathrm{~T}$ applied field, hence the easy plane description. They claim, then, that their $\mathrm{Nd}_{1.11} \mathrm{Fe}_{4} \mathrm{~B}_{4}$ exhibits magnetic anisotropy with an easy plane character and a four-fold symmetry and that this description is valid for all applied fields below 6 T. Surprisingly, given the extensive structural studies detailed above, there has been very little in the way of carefully examining the magnetic properties of $\mathrm{Nd}_{1+\epsilon} \mathrm{Fe}_{4} \mathrm{~B}_{4}$, with the noted exception of [5]. In addition, as far as we can tell, no results exist pertaining to the thermal properties or electrical transport properties of this compound.

Large single crystals of $\mathrm{Nd}_{1+\epsilon} \mathrm{Fe}_{4} \mathrm{~B}_{4}$ were grown via flux reaction for this study with the purpose of performing resistivity and heat capacity measurements; interesting features in the magnetization were later probed as well. These samples were large enough to shape by polishing which allows flexibility in removal of impurity phases by viewing the surfaces with scanning electron microscopy (SEM). This technique, when used along with isothermal, room-temperature $M(H)$ measurements, can result in an extremely phase pure sample. In our case, the Nd-rich flux reaction described below produces as-grown crystals with average $\mathrm{Nd}$ content approaching the maximum reported by [10]. These crystals yield observations of low temperature $M(H)$ behavior that does not mimic the almost step-function like curves described in [5] but rather increases more gradually with a previously unobserved behavior below applied field $0.7 \mathrm{~T}$ which has magnetization in the $\left[\begin{array}{lll}1 & 0 & 0\end{array}\right]$ direction that is larger than the magnetization at the same applied field in the $\left[\begin{array}{lll}1 & 1 & 0\end{array}\right]$ direction. Above $1 \mathrm{~T}$, however, the situation reverses and the maximum is in the [lll 110$]$ direction in agreement with [5]. Initial attempts to understand this behavior solely in terms of shape anisotropy have failed, suggesting that a more complex mechanism is responsible for this deviation from the previous description of [5]. We also observe, through resistivity, magnetization, and heat capacity measurements, a Curie temperature, $\mathrm{T}_{\mathrm{C}}$, of $7 \mathrm{~K}$. This is lower than previously reported values $[11,5]$. Finally, our heat capacity measurements up to $100 \mathrm{~K}$ show evidence of crystal field splitting.

\section{Experimental Details}

Single crystals of $\mathrm{Nd}_{1+\epsilon} \mathrm{Fe}_{4} \mathrm{~B}_{4}$ were grown via flux growth as described previously [12]. Crystals as large as $0.5 \times 0.5 \times 1 \mathrm{~cm}^{3}$ resulted with $a, b$, and $c$ faces present that could be verified by $\mathrm{x}$-ray diffraction (XRD) for orientation purposes. Single crystal XRD was performed on small flakes from these samples using a Rigaku 
difractometer with Dectris Pilatus 200K detector (Mo $K_{\alpha}, 0.71073 \AA$ ) and the structure was refined using SHELX [13]. Powder XRD was performed on ground samples using a PANalytical XPert Pro diffractometer (Cu $K_{\alpha 1}, 1.5406 \AA$ ) and relevant structure models were analyzed using the FULLPROF software package [14]. Elemental composition analysis was performed using a Hitachi TM-3000 electron microscope equipped with a Bruker Quantax 70 energy dispersive X-ray spectrometer (EDX) and using single crystals of $\mathrm{Nd}_{2} \mathrm{Fe}_{14} \mathrm{~B}$ as a comparative standard. EDX analysis on many different surfaces gives $\epsilon=0.16(8)$ and either does not contain the precision to to differentiate between previously reported values of $\epsilon$ or is probing some changes in $\epsilon$ or both. Prior experience with $\mathrm{Nd}_{2} \mathrm{Fe}_{14} \mathrm{~B}$ crystals shows the resolution limits in these experiments are at least one percent in Nd composition. Therefore we cannot simply use EDX to determine, say, the average $\epsilon$ for naming purposes of the samples here since the spread of values obtained from such measurements is larger than the spread of all values previously reported from diffraction measurements. However, these results are good enough to distinguish between $\mathrm{Nd}_{1+\epsilon} \mathrm{Fe}_{4} \mathrm{~B}_{4}$ and $\mathrm{Nd}_{2} \mathrm{Fe}_{14} \mathrm{~B}$ or any other impurity phases. As a result these measurements proved invaluable when fabricating samples because the surfaces could be examined for ferromagnetic impurities prior to magnetic characterization.

Magnetization measurements were performed using a Quantum Design Magnetic Property Measurement System (MPMS) for applied fields up to $5 \mathrm{~T}$ and temperatures between $4.2 \mathrm{~K}$ and $300 \mathrm{~K}$. The sample, shown in fig. 1, was either mounted to quartz glass paddles with marginally small amounts of cyanoacrylate for $M(H)$ and $\chi(T)$ measurements or mounted in the same way to a brass rotator whose axis of rotation is perpendicular to the applied field for measurements of the angular dependence of the magnetization. The magnetization component parallel to the applied field direction is the quantity measured here. We will actually call these angular dependent measurements $M(\theta)$ for all angles; specific geometries for each experiment will be specified as necessary. A field-dependent (constant susceptibility) diamagnetic background was subtracted from data collected on this rotator.

Resistivity measurements were done in a Quantum Design Physical Property Measurement System (PPMS) using the four-point technique with platinum wires for fields up to $8 \mathrm{~T}$ and temperatures between 2.5 $\mathrm{K}$ and $400 \mathrm{~K}$. Wires were attached to the sample using Dupont 4929N silver paste. The applied magnetic field was parallel to the $\left[\begin{array}{lll}0 & 1 & 0\end{array}\right]$ direction as labeled in fig. 1 .

Heat capacity measurements were performed using a

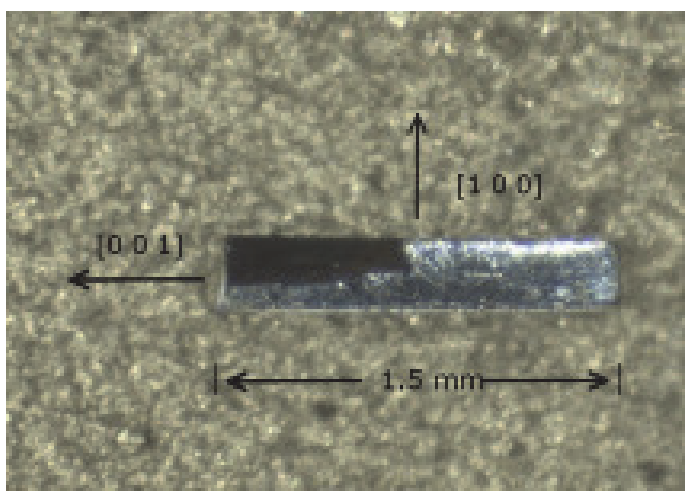

Figure 1: Photograph of the $\mathrm{Nd}_{1.143} \mathrm{Fe}_{4} \mathrm{~B}_{4}$ crystal used for the magnetization and resistivity measurements here. Its name will henceforth be "Sample 1".

Quantum Design PPMS for applied fields up to $8 \mathrm{~T}$ and temperatures between $2.5 \mathrm{~K}$ and $300 \mathrm{~K}$. These measurements were performed on as-grown single crystals with the applied field parallel to the $\left[\begin{array}{lll}1 & 0 & 0\end{array}\right]$ direction.

\section{Results and Discussion}

Figure 1 shows a photograph of the sample used for the magnetization and resistivity data shown in this study that will henceforth be referred to as "Sample 1". The sample was polished down to a $0.3 \times 0.2 \times 1.5 \mathrm{~mm}^{3}$ $\left(l_{a} \times l_{b} \times l_{c}\right)$ rectangular prism. Incidentally, for a perfect rectangular prism these dimensions correspond to demagnetization factors of $0.38,0.55$, and 0.07 respectively [15]. The sample in fig. 1 suffers from a large chip in one corner which can easily be seen in the photograph and which makes it not a perfect rectangular prism. Even if the sample was a perfect rectangular prism, it happens to have differing demagnetization factors along the principle axes which will inevitably provide some background effects in a $M(\theta)$ measurement. We will be particularly concerned with such background effects in magnetization measurements made by rotating through the $a-b$ plane which we will show has the most subtle features and it is, as a result, most important to reduce the shape anisotropy background in this plane. We have done this for "Sample 1" by attempting to make $l_{a}$ and $l_{b}$ as identical as possible so their respective demagnetization factors are nearly equal. If we were to instead make one of these values much larger than the other the opposite extreme could be realized. In such a plate-like sample the shape anisotropy background in a $M(\theta)$ measurement would then be maximal. This point will be demonstrated in more detail below. 


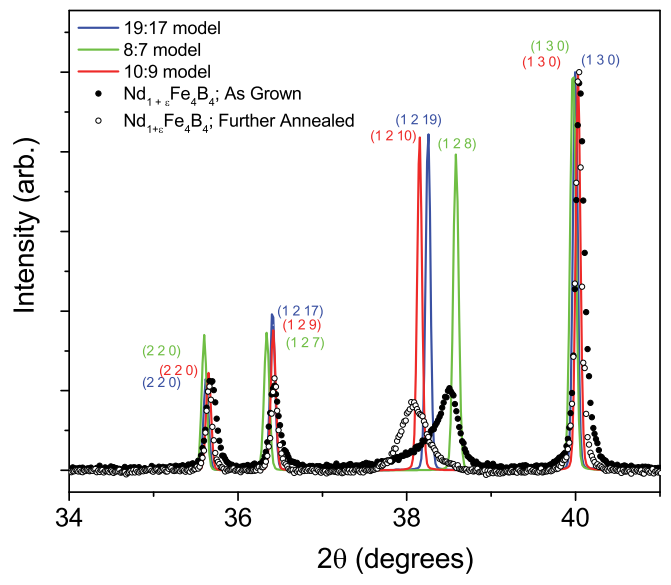

Figure 2: Solid data points: Background-subtracted powder $\mathrm{x}$-ray diffraction on ground single crystals of $\mathrm{Nd}_{1.143} \mathrm{Fe}_{4} \mathrm{~B}_{4}$ from the same batch as "Sample 1". The open circle points are that same powder after grinding and then annealing in a sealed silica tube at $500^{\circ} \mathrm{C}$. The solid lines represent simulations of PXRD patterns for different, commensurate superstructure models for comparison's sake.

Powder XRD measurements were performed on ground single crystals from the same batch as "Sample 1". The background-subtracted data are displayed with solid points in fig. 2. For the sake of comparison, several commensurate model simulations are also shown by solid lines and appear with (h k l) labels for their respective peaks. It is clear that no single, commensurate model fits the data well. The PXRD data from these asgrown, powdered crystals (solid points) in fig. 2 has a broad peak with a maximum at $38.6^{\circ}$. This maximum aligns with that of the $m: n=8: 7$ model. The peak has a tail, however, that indicates a multitude of local superstructures. Not surprisingly, the observed, broad peaks of this type span the $2 \theta$ values for the different simulations corresponding to the peaks with $(h k l)$ with $l=m$. This is clear evidence of variable stoichiometry in the bulk crystals and this evidence, along with evidence from the aforementioned prior studies, suggests that the inability to ascribe a single superstructure description is innate for large samples. All such broad peaks in the as-grown samples have a maximum and high $2 \theta$ edge that suggest a solubility limit near $\epsilon \approx \frac{1}{7}$ just as the broad peak at $38.6^{\circ}$ exemplifies. The $8: 7$ model was also the structure refined from single crystal XRD. The unit cell parameters of best fit determined from single crystal XRD were $a=7.130(3), b=7.130(3), c=27.358(9)$, $\alpha=\beta=\gamma=90^{\circ}$. This is a unit cell similar to that first reported by [1]. Single crystal XRD suggests that the atoms in our samples are arranged similarly to the scheme outlined in [9] but with the 8:7 ratio descrip- tion being more accurate than the 10:9 description; this fact is evidenced by our result for the $c$ lattice parameter. However, no single superstructure model could fit all observed intensities. We ultimately settled on the same space group, Pccn, as the authors of [9]. Our unit cell using the Pccn space group will then have four neodymium atoms as opposed to the five neodymium atoms of [9] and seven iron atoms as opposed to the nine iron atoms in [9]. These atoms are arranged within the unit cell exactly the same as demonstrated in [9] for the $a$ and $b$ atomic coordinates and the $c$ atomic coordinates are simply fractions of the unit cell corresponding to the best fit 8:7 ratio. This is exactly in accord with all prior reports and the reader is referred to the references for further structural detail as this problem has been exhaustively studied. It is, however, worth reiterating the point here that $m: n$ is linked to the unit cell of best fit from SCXRD measurements. Experiments on crystals, even crystals from the same batch, never completely agree on the best fit $c$ lattice parameter. Once a single unit cell is selected for refinement, spots that are not predicted for the selected model are observed, see [7] for example. The selection of a single unit cell here is an action that is at best an approximation for determining the most common value of some $m: n$ distribution function and is ultimately an invalid approach; it is however the best method at our disposal to understand the characteristics of $\epsilon$. With the inadequacies of the analysis in mind, we report that refinements in the selected structure produced fits with $R_{1}=0.08$. The model described here also fits the PXRD data of fig. 2 well, with the obvious exception of the $(h k l)$ with $l=m$ reflections. As the statistics of Reitveld refinements of the PXRD data were dominated by the shared peaks with $l \neq m$ and by the background, our choice of the value of $\epsilon$ for naming purposes here was not influenced by the PXRD data since the $\chi^{2}$ agreement values came out very close for all superstructure model refinements attempted. The differences in fits in single crystal XRD refinements were more stark so we used them to pick our best $\epsilon$ value for naming purposes here. As expected, some additional spots in the single crystal XRD not predicted by this 8:7 superstructure model were also present, an indication of the shortcomings of this description. Because the 8:7 commensurate structure model is that of best fit we will call our $\mathrm{Nd}_{1+\epsilon} \mathrm{Fe}_{4} \mathrm{~B}_{4}$ crystals from this study $\mathrm{Nd}_{1.143} \mathrm{Fe}_{4} \mathrm{~B}_{4}$ with the understanding that $\epsilon=0.143$ is representative only of the best fit commensurate model behavior. The large value of $\epsilon$ here seems reasonable considering the slow formation conditions in a $\mathrm{Nd}$ flux. The $\epsilon$ distribution can then be altered by annealing in a Nd-poor environment. The open circle points in fig. 2 
represent PXRD data taken on as-grown samples after grinding then further annealing at $500^{\circ} \mathrm{C}$ in an evacuated quartz ampoule. The $\epsilon$ distribution has clearly shifted towards a smaller $m: n$ ratio. We would expect then, in order to conserve $\mathrm{Nd}$, that we might be able to observe elemental $\mathrm{Nd}$ peaks or peaks from $\mathrm{Nd}-\mathrm{O}$ phases in the open circle data but this was not the case practically. The aforementioned ferromagnetic impurity phases that exist in the as grown crystals, observable on surfaces in SEM-EDX measurements, were also not prominent enough to be observed in the PXRD data of fig. 2.

Figure 3 plots the results of magnetic susceptibility measurements on "Sample 1" at an applied field of 0.1 $\mathrm{T}$ parallel to the $\left[\begin{array}{lll}0 & 1 & 0\end{array}\right]$ direction as defined in fig. 1. The small value of $\chi$ at higher temperatures is clear evidence of a ferromagnetic impurity-free sample. The inset shows the reciprocal of that quantity and the result clearly shows paramagnetic behavior above a ferromagnetic transition temperature of $7 \mathrm{~K}$.

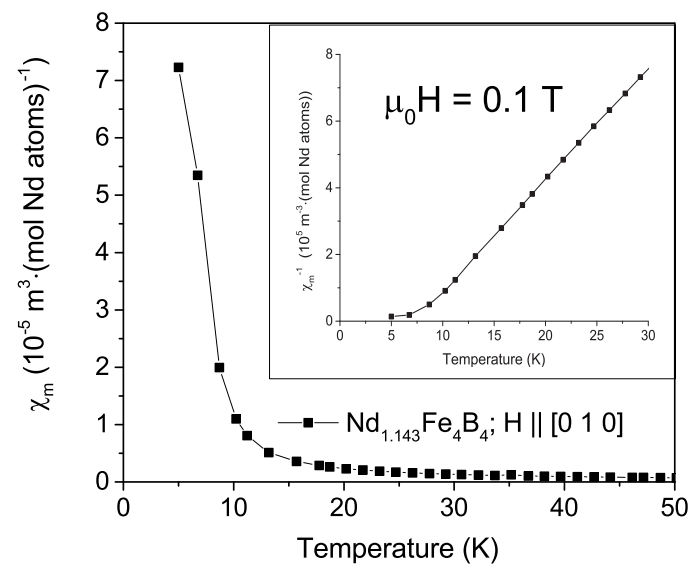

Figure 3: Molar magnetic susceptibility for "Sample 1". The reciprocal of that quantity is shown in the inset.

Figure 4 displays a plot proportional to the square root of the quantity constructed by multiplying the temperature and the molar magnetic susceptibility. The data were collected at $5 \mathrm{~T}$. The units in fig. 4 are such that the $\mathrm{y}$-axis yields the effective magnetic moment per $\mathrm{Nd}$ atom, $\mu_{e f f}$, determined from the Curie constant in the Curie-Weiss law. A Curie-Weiss curve with a Weiss temperature of $7 \mathrm{~K}$ and $\mu_{e f f}=4.5 \mu_{B}$ is shown by a dashed line and clearly the $\mathrm{Nd}_{1.143} \mathrm{Fe}_{4} \mathrm{~B}_{4}$ sample here obeys the Curie-Weiss law well. At higher temperature this type of Curie-Weiss plot will become constant and yield the effective moment, here $4.5 \mu_{B}$ per $\mathrm{Nd}$ atom. This number for $\mu_{e f f}$ differs from the value of $3.8 \mu_{B}$ reported in [5]. The value from [5] is closer to the free ion

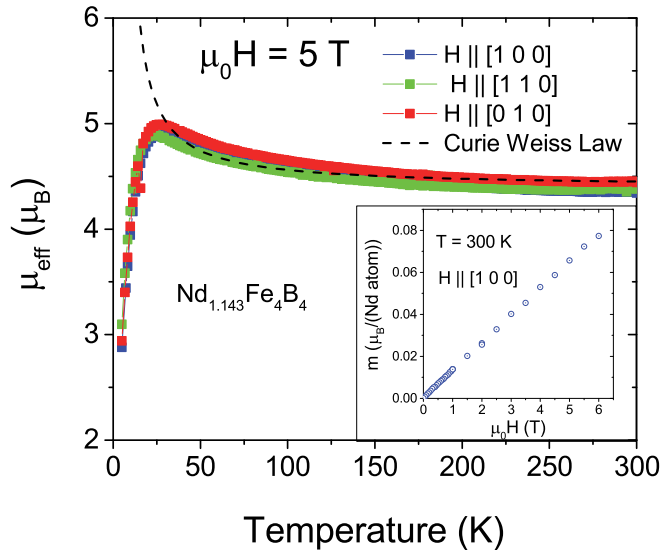

Figure 4: $\mu_{e f f}$ per $\mathrm{Nd}$ atom in "Sample 1" as determined from the Curie constant. $\mu_{e f f}$ is calculated by $\mu_{e f f}=$ $\left(3 k_{B} \cdot T \cdot \chi_{m}\right)^{1 / 2}\left(N_{A} \cdot \mu_{0}\right)^{-1 / 2}\left(\mu_{B}\right)^{-1}$. The dashed line is a CurieWeiss law simulation with a Weiss temperature of $7 \mathrm{~K}$ and $\mu_{\text {eff }}=4.5$ $\mu_{B}$. The inset shows the magnetization dependence of the sample on applied field at room temperature.

value of $3.62 \mu_{B}$ than our value here; we do not have an explanation for the discrepancy at this time. The room temperature $M(H)$ curve in the inset of fig. 4 is especially telling as to the high quality of "Sample 1". As $\mathrm{Nd}_{1.143} \mathrm{Fe}_{4} \mathrm{~B}_{4}$ is in the paramagnetic regime here, this plot ought to be linear with a zero intercept for a sample that contains no ferromagnetic impurities. Confirming such behavior is essential before proceeding to measure other magnetic properties as impurities in $\mathrm{Nd}_{1+\epsilon} \mathrm{Fe}_{4} \mathrm{~B}_{4}$ are likely strong magnets themselves. Removing such impurities can be a labor intensive process and samples were usually, as in the case of "Sample 1", very small $(\approx 1 \mathrm{mg}$ ) by the time they are acceptable. The small size of the samples is the reason that susceptibility measurements with the field parallel to the [ll $\left.0 \begin{array}{ll}0 & 1\end{array}\right]$ axis are not shown; reproducibility of magnetization experiments is often difficult in small moment samples and the magnetization is especially small in that direction at higher temperatures as shown originally in [5]. The qualitative behavior observed in [5] is, however, observed here, namely a sizable difference of the Weiss temperatures determined from magnetization measurements in the $[0$ 0 1] direction and from measurements in the basal plane. Such an anisotropy has been generally shown to be producible by crystal field splitting effects [16].

Figure 5(a) shows $M(H)$ curves with the field applied along different crystallographic directions at 4.2 K for "Sample 1". The solid points represent data collected with increasing and decreasing external applied field parallel to the $\left[\begin{array}{lll}1 & 1 & 0\end{array}\right]$ direction. No hysteresis 

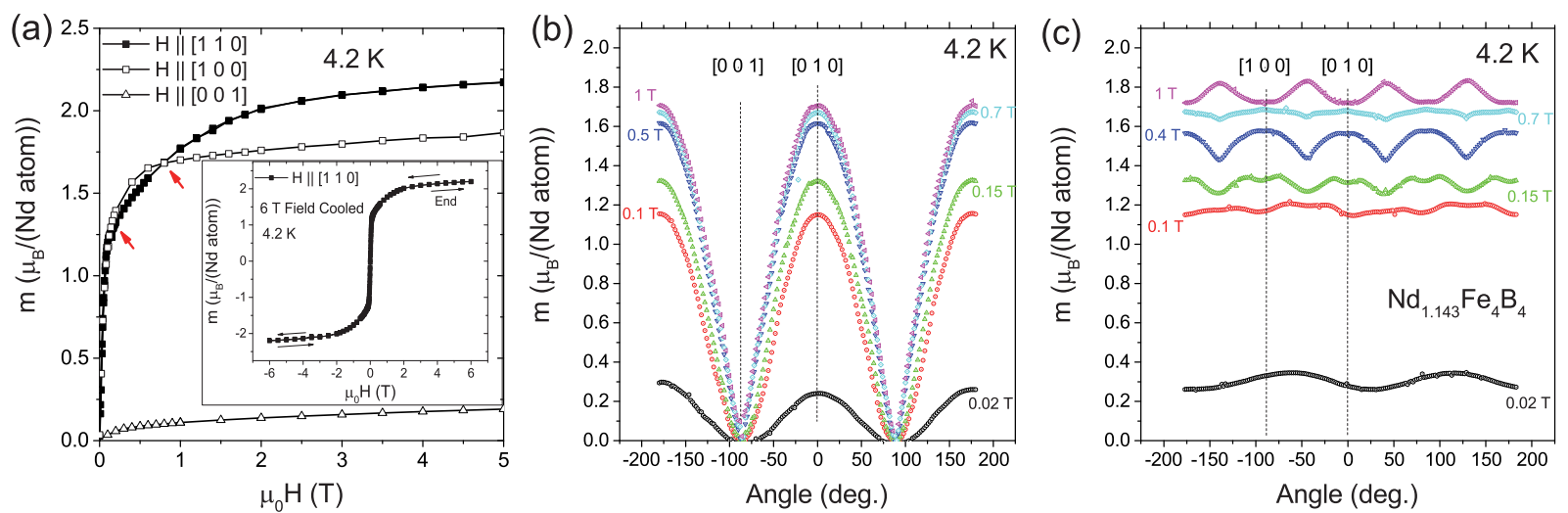

Figure 5: (a) The low temperature field dependence of the magnetization for the $\mathrm{Nd}_{1.143} \mathrm{Fe}_{4} \mathrm{~B}_{4}$ sample named "Sample 1". The field is parallel to the $\left[\begin{array}{lll}1 & 0 & 0\end{array}\right]$ direction for the open-square data points and is parallel to the $\left[\begin{array}{lll}1 & 1 & 0\end{array}\right]$ direction for the solid-square data points and is parallel to the [0 0 1] direction for the open-triangle data points. All solid-square data is actually part of the hysteresis curve shown in the inset and the points lie nearly exactly on top of one another showing no irreproducibility or coercivity whatsoever. (b) $b$ - $c$ plane magnetization as a function of sample angle with respect to field. (c) $a-b$ plane magnetization behavior. Shape anisotropy effects are the likely cause of the angular dependence of the 0.02 T curve.

is observed in this direction and the curves were completely reproducible under field-cooled or zero-fieldcooled conditions. This observation is in contrast to the irreversibility reported in [5] below $0.3 \mathrm{~T}$. Below $\approx 0.1$ $\mathrm{T}$ (marked by an arrow in fig. 5(a)) shape anisotropy is the dominant demagnetization mechanism in the crystal. Although $M(H)$ in this region varies between differently shaped samples, it is completely reproducible in a given sample regardless of history. Between $0.1 \mathrm{~T}$ and $0.7 \mathrm{~T}$ is previously unobserved behavior where the

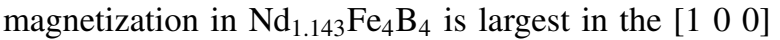
direction. $M(H)$ is reproducible no matter what the history in this region as well. Above $0.7 \mathrm{~T}$ our data are in good agreement with [5]. It is unknown to us whether the larger $\mathrm{Nd}$ content of our samples $(\epsilon=0.143)$ compared to the previous report $(\epsilon=0.111)[5]$ is important for this observation of an easy direction change. $M(H)$ with the applied field parallel to the [lllll $\left.\begin{array}{lll}0 & 1\end{array}\right]$ direction is also displayed and the large planar anisotropy is evident here just as it was in [5].

Figure 5(b) displays $M(\theta)$ at $4.2 \mathrm{~K}$ while rotating "Sample 1" in the $b$-c plane from [0 $\left.\begin{array}{lll}0 & 1 & 0\end{array}\right]$ to $\left[\begin{array}{lll}0 & 0 & 1\end{array}\right]$. Here $M(\theta)$ resembles the absolute value of a cosine function and approaches a node at $90^{\circ}$ when the basal plane moment has no projection along the field direction unit vector. At $45^{\circ}$ the magnetization is simply the maximum value divided by $\sqrt{2}$. This is precisely the expected behavior for a uniaxial ferromagnet rotated in any plane containing the two easy directions or for an easy $a-b$ plane magnet rotated in a plane containing the $c$ axis, a behavior observed here in the $b-c$ plane for all applied fields up to $5 \mathrm{~T}$. Figure $5(\mathrm{c})$ displays $M(\theta)$ at $4.2 \mathrm{~K}$ while rotating in the $a-b$ plane from [ $\left[\begin{array}{lll}0 & 1 & 0\end{array}\right]$ to $\left[\begin{array}{lll}1 & 0 & 0\end{array}\right]$. For a sample with four easy directions in the basal plane as described in [5], as the sample rotates from the [lll 110$]$ axis to the [llll $\left.\begin{array}{lll}0 & 1 & 0\end{array}\right]$ or $\left[\begin{array}{lll}1 & 0 & 0\end{array}\right]$ direction $M(\theta)$ ought to decrease and the function should look like a trigonometric function. However, the periodicity should be different than the case represented by the rotation of fig. 5(b). The appropriate function will now consist of repeating piecewise sine functions on the full $360^{\circ}$ domain of $\theta$; each repeating piecewise function has a width of only $90^{\circ}$. The maximum of each piecewise sine function is centered within its $90^{\circ}$ window and the minima over the full $360^{\circ}$ range of $\theta$ will occur at the [ $\left.\begin{array}{lll}1 & 0 & 0\end{array}\right],\left[\begin{array}{lll}0 & 1 & 0\end{array}\right],\left[\begin{array}{ll}-1 & 0\end{array}\right.$ 0 0], and [0-1 0] directions. Our observations at $1 \mathrm{~T}$ and above qualitatively resemble this behavior. Also, the maxima and minima of each of the fig. 5(c) curves agree well with the field sweep values of fig. 5(a) as expected. The reader will notice that these maxima and minima are never exactly related to each other by a factor of $\sqrt{2}$; the $\left[\begin{array}{lll}1 & 0 & 0\end{array}\right]$ direction maximum is always larger than expected, by $\approx 10 \%$ or more. This is also nearly the case for the moments given in [5]. This deviation could be a sign of some kind of complicated moment canting or, perhaps, some even more complicated effect. As the applied field is decreased the $M(\theta)$ curves qualitatively change. At $0.7 \mathrm{~T} M(\theta)$ appears almost flat, as if there is no longer an in-plane anisotropy effect. At $0.4 \mathrm{~T}$ the symmetry is clearly different, with the $\left[\begin{array}{lll}1 & 0 & 0\end{array}\right]$ direction now appearing as an easy direction of four-fold symmetry. Below this applied field value the $M(\theta)$ behavior is 


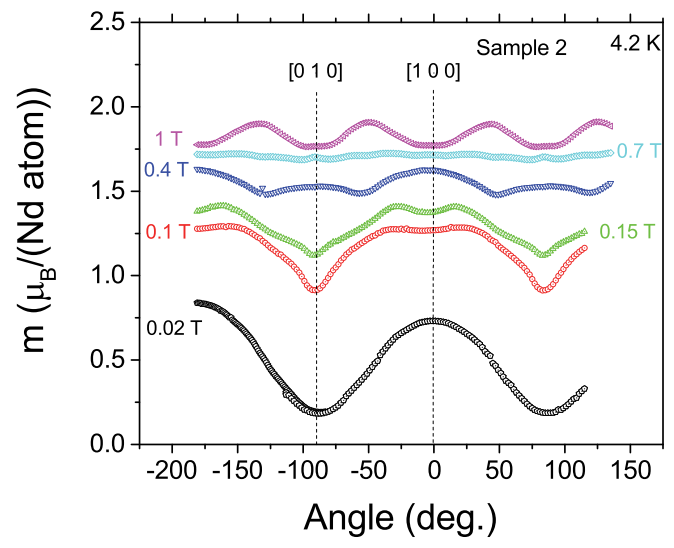

Figure 6: The angular dependence of a plate-like sample, "Sample 2", for comparison with the sample of fig. 5, "Sample 1". Though the demagnetization effects are much more prominent here, the easy axis change between $0.4 \mathrm{~T}$ and $1 \mathrm{~T}$ is still evident.

more complicated.

The reader may question if shape anisotropy effects, which can be significant at low fields, could be responsible for the easy direction change observed at $0.7 \mathrm{~T}$. We attempt to address this issue experimentally by introducing a second sample that is plate-like in shape called "Sample 2". The dimensions of "Sample 2" are roughly $0.7 \times 0.05 \times 1.6 \mathrm{~mm}^{3}\left(l_{a} \times l_{b} \times l_{c}\right)$ and correspond to demagnetization factors of $0.09,0.88$, and 0.03 respectively. The $M(\theta)$ data for "Sample 2" is displayed in fig. 6. The differences in the $0.02 \mathrm{~T} M(\theta)$ curves of fig. 5(c) and fig. 6 are immediately apparent. "Sample 2" clearly has a sinusoidal-like signal whose importance diminishes as the applied field is increased. This is a reasonable result from a sample with a large discrepancy in demagnetization factors. However, the easy direction change at $0.7 \mathrm{~T}$ is still apparent and the curves below $0.7 \mathrm{~T}$ resemble those of fig. 5(c) with an additional background attributed here to large shape anisotropy. Both samples were created in the same way and are assumed to have identical $\epsilon$ distributions. If the observation of this easy direction change is only made possible by having $\epsilon$ near the solubility limit then the differences in the $M(H)$ curves shown here and in [5] might be explicable. If, however, this easy direction change behavior is robust for all $\epsilon$ in the previously observed range then it ought to have been observed in [5]. Future experiments on spherically shaped samples with different average values of $\epsilon$ could help provide interesting further evidence to resolve this issue. Theoretical treatment of the problem may also prove enlightening.

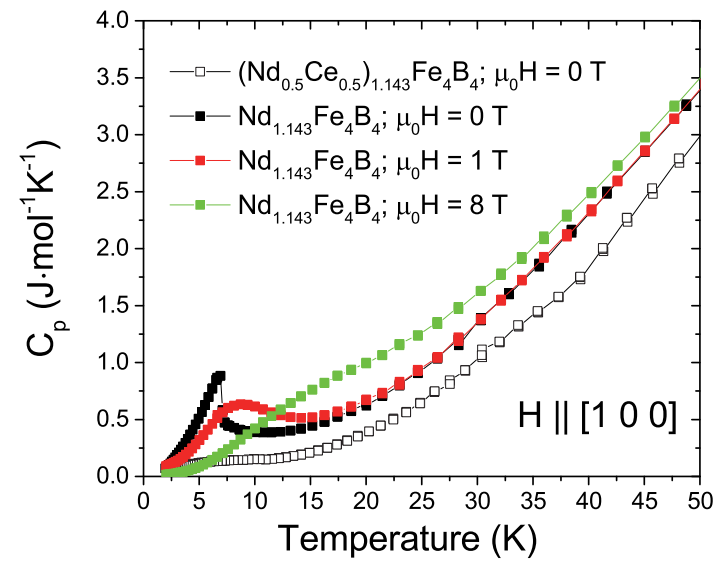

Figure 7: The low temperature heat capacity of a $\mathrm{Nd}_{1.143} \mathrm{Fe}_{4} \mathrm{~B}_{4}$ crystal compared to a crystal of $\left(\mathrm{Nd}_{0.5} \mathrm{Ce}_{0.5}\right)_{1+\epsilon} \mathrm{Fe}_{4} \mathrm{~B}_{4}$.

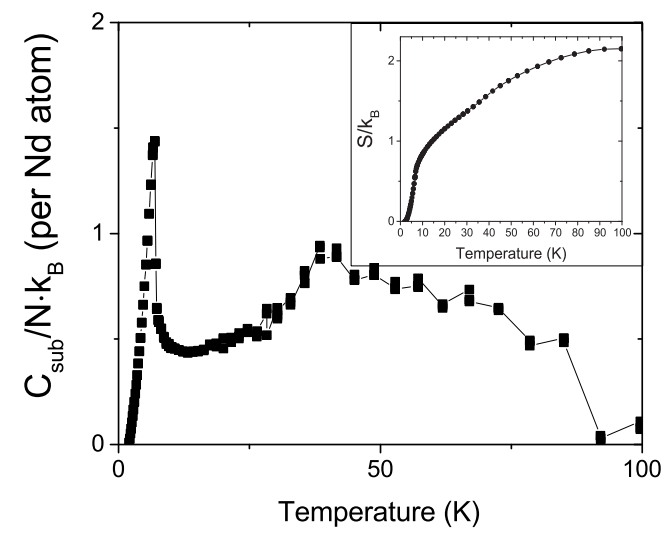

Figure 8: The subtraction of the zero field curves of fig. 7 is displayed and scaled so that the curve represents the contribution from the remaining $\mathrm{Nd}$ atoms. The scaling is done by multiplying by $16 / \mathrm{R}$. The inset shows the entropy as a function of temperature. 


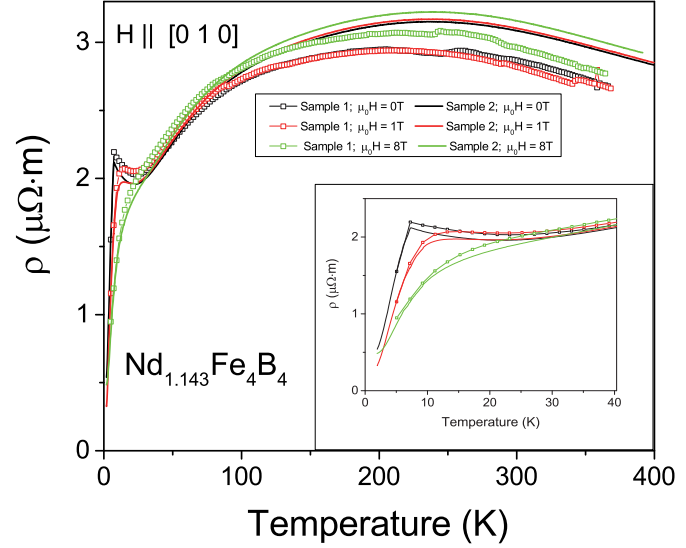

Figure 9: The resistivity along the [0 001 l] direction of "Sample 1" and "Sample 2".

The heat capacity, $C_{p}$, was measured on a larger $\mathrm{Nd}_{1.143} \mathrm{Fe}_{4} \mathrm{~B}_{4}$ crystal $(\approx 12 \mathrm{mg}$ ) than "Sample 1" to reduce data scatter, though all results shown here in fig. 7 are consistent with measurements also performed on "Sample 1". The magnetic impurities associated with this large sample did not require removal for $C_{p}$ measurements. The ferromagnetic transition at zero field is apparent and the Curie temperature is once again observed around $7 \mathrm{~K}$. As expected, we observe the smearing out of the feature associated with this transition as field is increased. We also observe field independence of $C_{p}$ above $50 \mathrm{~K}$. The heat capacity of a sample made with half of the $\mathrm{Nd}$ atoms replaced by $\mathrm{Ce}$ atoms was also measured and is displayed in fig. 7. Magnetization measurements indicate no long-range magnetic ordering transition in this isostructural $\left(\mathrm{Nd}_{0.5} \mathrm{Ce}_{0.5}\right)_{1.143} \mathrm{Fe}_{4} \mathrm{~B}_{4}$ phase. The $\left(\mathrm{Nd}_{0.5} \mathrm{Ce}_{0.5}\right)_{1.143} \mathrm{Fe}_{4} \mathrm{~B}_{4}$ sample can, then, be used as a kind of lattice standard to help roughly estimate the magnetic contribution to the heat capacity of $\mathrm{Nd}_{1.143} \mathrm{Fe}_{4} \mathrm{~B}_{4}$. For this estimate to be valid the $\left(\mathrm{Nd}_{0.5} \mathrm{Ce}_{0.5}\right)_{1.143} \mathrm{Fe}_{4} \mathrm{~B}_{4}$ crystals must be isostructural to the $\mathrm{Nd}_{1.143} \mathrm{Fe}_{4} \mathrm{~B}_{4}$ crystals. The isostructural nature of $\left(\mathrm{Nd}_{0.5} \mathrm{Ce}_{0.5}\right)_{1.143} \mathrm{Fe}_{4} \mathrm{~B}_{4}$ is verified here by two methods. XRD experiments off naturally forming crystal facets of $\left(\mathrm{Nd}_{0.5} \mathrm{Ce}_{0.5}\right)_{1.143} \mathrm{Fe}_{4} \mathrm{~B}_{4}$ produce peaks that are always consistent with the undoped counterpart. These crystals have also been ground and the PXRD patterns are nearly identical to the as-grown data displayed in fig. 2 save the slight shift of all peaks due to a small volume contraction $(a=7.109(4), c=27.342(9))$. SEMEDX measurements on different spots give the value of $\epsilon \approx 0.15(7)$. As with the undoped sample, we will use the name associated with $\epsilon=0.143$ to describe the doped sample because of the similarities in flux growth conditions and the similar skew of the $l=m$ PXRD peaks (fig. 10). As evidenced by fig. 7, the Ce-doped sample has a significantly reduced heat capacity compared to $\mathrm{Nd}_{1.143} \mathrm{Fe}_{4} \mathrm{~B}_{4}$. In fig. 8, a plot of the difference of $C_{p}$ between $\mathrm{Nd}_{1.143} \mathrm{Fe}_{4} \mathrm{~B}_{4}$ and $\left(\mathrm{Nd}_{0.5} \mathrm{Ce}_{0.5}\right)_{1.143} \mathrm{Fe}_{4} \mathrm{~B}_{4}$ shows that this difference persists to $100 \mathrm{~K}$. The units of fig. 8 are different than those of fig. 7; here the plot shows the heat capacity per remaining $\mathrm{Nd}$ atom after the subtraction. If the ground-state energy levels of $\mathrm{Nd}$ atoms in $\mathrm{Nd}_{1.143} \mathrm{Fe}_{4} \mathrm{~B}_{4}$ and $\left(\mathrm{Nd}_{0.5} \mathrm{Ce}_{0.5}\right)_{1.143} \mathrm{Fe}_{4} \mathrm{~B}_{4}$ are split qualitatively similarly then the total ground state entropy per $\mathrm{Nd}$ atom can be obtained from this procedure, however its temperature dependence will not be exact. Interestingly, we obtain an entropy, $S / k_{B}$, of 0.63 below $\mathrm{T}_{\mathrm{C}}$, which is nearly $\ln (2)$, and an entropy of 2.1 , which is nearly $\ln (10)$, over the entire range in fig. 8 . This is the result one expects from a $\mathrm{Nd}^{3+}$ ion with a ${ }^{4} I_{9 / 2}$ ground state and a relatively low $\mathrm{T}_{\mathrm{C}}$. Since this type of analysis involves several assumptions and is sensitive to small errors in the sample masses, the excellent agreement is possibly fortuitous. The shape of this curve above $7 \mathrm{~K}$ is good evidence of crystal field splitting of the ${ }^{4} I_{9 / 2}$ level; one sees the low temperature feature associated with the ferromagnetic transition and an additional feature reminiscent of a simple Schottky peak with a maximum near $40 \mathrm{~K}$. Such a broad peak can be expected qualitative behavior even for a system of more than two states, see [17] for example. We are not the first to suggest strong crystal field splitting effects in $\mathrm{Nd}_{1+\epsilon} \mathrm{Fe}_{4} \mathrm{~B}_{4}$; the effect was already suggested to be important by the anisotropic susceptibility measurements in [5]. Since the low temperature heat capacity data from both samples are not described by a simple Debye model plus a linear electronic contribution, the $C_{p}$ vs. $T$ data at higher temepratures $(20 \mathrm{~K}-300 \mathrm{~K})$ were fit to a Debye function yielding an approximate value of $540 \mathrm{~K}$ for the Debye temperature. However, strict adherence of the heat capacity to a Debye model in this range is not observed.

We performed resistivity measurements as a function of temperature, $\rho(T)$, along the [ $\left[\begin{array}{lll}0 & 0 & 1\end{array}\right]$ direction on both "Sample 1" and "Sample 2" and the results are displayed in fig. 9 and the curves for both samples are in reasonable accord with one another. $\rho$ is slightly higher than that of a normal metal at room temperature. Data near the ferromagnetic transition are displayed in the inset. As expected, the resistivity decreases dramatically as temperature is reduced below $\mathrm{T}_{\mathrm{C}}$ and as the applied field is increased this feature near $7 \mathrm{~K}$ is smeared out. On cooling from $25 \mathrm{~K}$ to $7 \mathrm{~K}$ we see an upturn that is likely associated with enhanced scattering due to the on- 


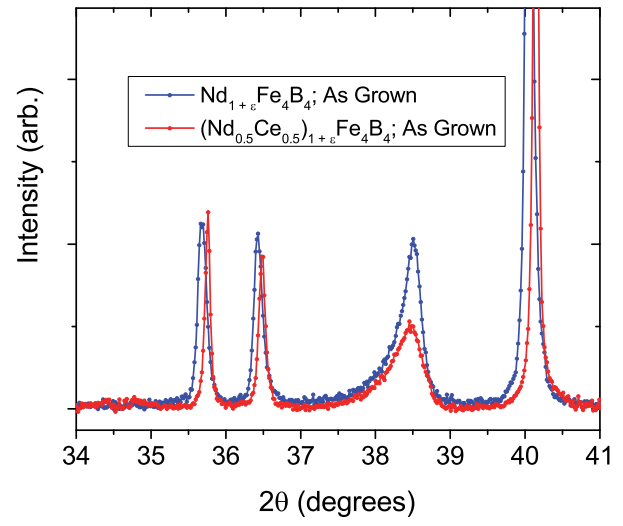

Figure 10: Blue data points are background-subtracted powder $\mathrm{x}$ ray diffraction on ground single crystals of $\mathrm{Nd}_{1.143} \mathrm{Fe}_{4} \mathrm{~B}_{4}$ from the same batch as "Sample 1". Red data points are backgroundsubtracted powder $\mathrm{x}$-ray diffraction on ground single crystals of $\left(\mathrm{Nd}_{0.5} \mathrm{Ce}_{0.5}\right)_{1.143} \mathrm{Fe}_{4} \mathrm{~B}_{4}$.

set of fluctuations as the ferromagnetic transition is approached. The most interesting feature in the $\rho(T)$ data is the broad maximum near $200 \mathrm{~K}$, which is very unusual for a typical metal. A broad maximum has been observed before in systems with short range magnetic order or strong magnetic fluctuations. For example, the system $\mathrm{Fe}_{2} \mathrm{P}$ is a well known case where a maximum in $\rho(T)$ is observed at temperatures above a Curie temperature [18] which is verified by neutron diffraction experiments to be caused by short-range ordering [19] of iron moments. Though we do not claim such a pronounced, short-range ordering effect as is observed in $\mathrm{Fe}_{2} \mathrm{P}$, we do suspect that magnetic correlations among the iron moments in $\mathrm{Nd}_{1.143} \mathrm{Fe}_{4} \mathrm{~B}_{4}$ are responsible for this enhanced scattering. At temperatures above $100 \mathrm{~K}$ the magnetoresistance is positive with a value of 2 to $3 \%$ for an applied field of $8 \mathrm{~T}$, which is also suggestive of magnetic fluctuations.

\section{Summary}

We have carried out heat capacity and resistivity measurements on a $\mathrm{Nd}_{1+\epsilon} \mathrm{Fe}_{4} \mathrm{~B}_{4}$ crystal with $\epsilon$ near the solubility limit of $\frac{1}{7}$. Comparison of the heat capacity data of $\mathrm{Nd}_{1.143} \mathrm{Fe}_{4} \mathrm{~B}_{4}$ and $\left(\mathrm{Nd}_{0.5} \mathrm{Ce}_{0.5}\right)_{1.143} \mathrm{Fe}_{4} \mathrm{~B}_{4}$ provide further evidence that the low-temperature ferromagnetism is, in fact, associated with $\mathrm{Nd}$ moments. Future magnetization measurements on $\left(\mathrm{Nd}_{0.5} \mathrm{Ce}_{0.5}\right)_{1+\epsilon} \mathrm{Fe}_{4} \mathrm{~B}_{4}$ samples without impurity phases could provide further evidence for this. Above $\mathrm{T}_{\mathrm{C}}$, differences in the heat capacity of $\mathrm{Nd}_{1.143} \mathrm{Fe}_{4} \mathrm{~B}_{4}$ and $\left(\mathrm{Nd}_{0.5} \mathrm{Ce}_{0.5}\right)_{1.143} \mathrm{Fe}_{4} \mathrm{~B}_{4}$ are dis- played and the difference in heat capacity of the former with respect to the latter is argued to be due to crystal field splitting effects, in reasonable accord with the anisotropic magnetic susceptibility observations of Givord et al. in [5]. The observation of a maximum in the resistivity of $\mathrm{Nd}_{1.143} \mathrm{Fe}_{4} \mathrm{~B}_{4}$ above $200 \mathrm{~K}$ is reported here and the claim is made that this effect is likely a short-range effect due to iron moments.

We also show evidence, from angular dependent magnetization measurements at fields larger than $0.7 \mathrm{~T}$ and up to $5 \mathrm{~T}$, that $\mathrm{Nd}_{1.143} \mathrm{Fe}_{4} \mathrm{~B}_{4}$ does indeed behave as a ferromagnet of substantial easy plane anisotropy and also with easy directions of four-fold symmetry in the crystallographic [ $\left[\begin{array}{lll}1 & 1 & 0\end{array}\right]$, etc. directions. We see behavior between $0.1 \mathrm{~T}$ and $0.7 \mathrm{~T}$ in these measurements that appears to be evidence that $\mathrm{Nd}_{1.143} \mathrm{Fe}_{4} \mathrm{~B}_{4}$ favors new easy directions, again of four-fold symmetry, in the [1 00 ], etc. directions at these intermediate applied fields and we show evidence that these features are present in two samples with extremely different shapes. Angular dependent studies like this one on samples with measurably different $\epsilon$ distributions might make interesting future experiments. In general, both experimental and theoretical description of the $\mathrm{Nd}_{1+\epsilon} \mathrm{Fe}_{4} \mathrm{~B}_{4}$ system is sparse and we hope that the measurements presented here will spur future interest.

\section{Acknowledgments}

Research sponsored by the Critical Materials Institute, an Energy Innovation Hub funded by the US Department of Energy, Office of Energy Efficiency and Renewable Energy, Advanced Manufacturing Office (B.S.C., B.C.S.) and the Department of Energy, Office of Science, Basic Energy Sciences, Materials Sciences and Engineering Division (M.A.S, P.J.K., A.F.M., M.A.M., J.Q.Y.).

\section{References}

[1] N. F. Chaban, Yu. B. Kuz'ma, N. S. Byilonyizhko, O. O. Kachmar, and N. B. Petryiv. Ternary [Nd, Sm, Gd]-Fe-B systems. Dopovidi Akademii Nauk Ukrains'koj RSR. Seriya A, FizikoTekhnichni ta Matematichni Nauki, 11(16):873-876, 1979.

[2] H. F. Braun, M. Pilizzone, and K. Yvon. Ferromagnetic borides with incommensurate rare earth and iron sublattices: $\mathrm{R}_{1+\epsilon} \mathrm{Fe}_{4} \mathrm{~B}_{4}$. Proceedings of the 7 th international conference on solid compounds of transition elements, Grenoble, June 1982.

[3] K. Oesterreicher and H. Oesterreicher. On the phase $\mathrm{NdFe}_{4} \mathrm{~B}_{4}$ and its implication in the magnetic hardening of $\mathrm{Nd}_{2} \mathrm{Fe}_{14} \mathrm{~B}$ permanent magnets. J. Less-Common Met., 104(2):19-21, 1984.

[4] A. Bezinge, H. F. Braun, J. Muller, and K. Yvon. Tetragonal rare earth $(\mathrm{R})$ iron borides, $\mathrm{R}_{1+\epsilon} \mathrm{Fe}_{4} \mathrm{~B}_{4}(\epsilon \approx 0.1)$, with incommensurate rare earth and iron substructures. Solid State Commun., 55(2):131-135, 1985. 
[5] D. Givord, J. M. Moreau, and P. Tenaud. $\mathrm{Nd}_{5} \mathrm{Fe}_{18} \mathrm{~B}_{18}$ $\left(\mathrm{Nd}_{1.11} \mathrm{Fe}_{4} \mathrm{~B}_{4}\right)$, a new Nowotny-like phase. Structural and magnetic properties. Solid State Commun., 55(4):303-306, 1985.

[6] K. Oesterreicher and H. Oesterreicher. Nowotny chimney ladder compounds in $\mathrm{Nd}_{1+\epsilon} \mathrm{Fe}_{4-\mathrm{x}} \mathrm{Co}_{\mathrm{x}} \mathrm{B}_{4}$. Journal of Solid State Chemistry, 70(2):313-317, October 1987.

[7] A. Bezinge, H. F. Braun, and J. Muller. $\mathrm{Nd}_{1+\epsilon} \mathrm{Fe}_{4} \mathrm{~B}_{4}$ : A composition-modulated compound with incommensurate composite crystal structure. Phys. Rev. B, 36(3):1406-1414, 1987.

[8] G. Bocelli, G. Calestani, and F. Leccabue. Structural investigation of incommensurate $\mathrm{Nd}_{1+\epsilon} \mathrm{Fe}_{4} \mathrm{~B}_{4}$ phase. Solid State Commun., 65(11):1301-1305, March 1988.

[9] D. Givord, P. Tenaud, and J. M. Moreau. Refinement of the crystal structure of $\mathrm{R}_{1+\epsilon} \mathrm{Fe}_{4} \mathrm{~B}_{4}$ compounds $(\mathrm{R}=\mathrm{Nd}, \mathrm{Gd})$. Journal of the Less Common Metals, 123(1-2):109-116, 1986.

[10] S. C. Wang and Y. Li. A new structure of $\mathrm{Nd}_{1+\epsilon} \mathrm{Fe}_{4} \mathrm{~B}_{4}$ phase in NdFeB magnet. Journal of Materials Science, 40(14):3853$3855,2005$.

[11] K. H. J. Buschow, D. B. De Mooij, J. L. C. Daams, and H. M. van Noort. Phase relationships, magnetic and crystallographic properties of NdFeB alloys. Journal of the Less Common Metals, 115(2):357-366, 1986.

[12] M. A. Susner, B. S. Conner, B. I. Saparov, M. A. McGuire, G. M. Veith, E. J. Crumlin, H. B. Cao, B. C. Chakoumakos, and B. C. Sales. Growth and characterization of Ce-substituted $\mathrm{Nd}_{2} \mathrm{Fe}_{14} \mathrm{~B}$ single crystals. J. Magn. Magn. , In Press, doi: http://dx.doi.org/10.1016/j.jmmm.2016.10.127, 2016.

[13] G. M. Sheldrick. A Short History of SHELX. Acta Crystallographica Section A, 64(1):112-122, 2008.

[14] J. Rodriguez-Carvajal. Recent advances in magnetic structure determination by neutron powder diffraction. Physica B, 192(12):55 - 69, 1993.

[15] A. Aharoni. Demagnetizing factors for rectangular ferromagnetic prisms. Journal of Applied Physics, 83(6):3432, 1998.

[16] Y.-L. Wang. Crystal-field effects of paramagnetic Curie temperature. Physics Letters, 35A(5), 1971.

[17] A. Szytula, D. Kaczorowski, L. Gondek, A. Arulraj, S. Baran, and B. Penc. Magnetic properties of $\mathrm{NdAu}_{2} \mathrm{Ge}_{2}$. J. Magn. Magn. Mat., 321:3402, 2009.

[18] H. Fujii, T. Hōkabe, T. Kamigaichi, and T. Okamoto. Magnetic Properties of $\mathrm{Fe}_{2} \mathrm{P}$ Single Crystal. J. Phys. Soc. Jpn., 43(1):41, 1977.

[19] H. Fujii, Y. Uwatoko, K. Motoya, Y. Ito, and T. Okamoto. Neutron Scattering Investigation of Itenerant Electron System $\mathrm{Fe}_{2} \mathrm{P}$. J. Phys. Soc. Jpn., 57(6):2143, 1988. 